Effect Analysis Using Nonlinear Structural Equation Mixture Modeling

\author{
Axel Mayer \\ RWTH Aachen University \\ Nora Umbach \\ University of Tübingen \\ Barbara Flunger \\ Utrecht University \\ Augustin Kelava \\ University of Tübingen
}

This is a pre-copyedited, author-produced PDF of an article published in Structural Equation Modeling: A Multidisciplinary Journal following peer review.

\begin{abstract}
Citation:
Mayer, A., Umbach, N., Flunger, B., \& Kelava, A. (2017) Effect analysis using nonlinear structural equation mixture modeling, Structural Equation Modeling, 24, 556-570, doi:10.1080/10705511.2016.1273780
\end{abstract}

Author Note

Axel Mayer, RWTH Aachen University; Nora Umbach, University of Tübingen; Barbara Flunger, Utrecht University; Augustin Kelava, University of Tübingen.

Correspondence concerning this article should be addressed to Axel Mayer, Department of Psychological Methods, Institute of Psychology, RWTH Aachen University, Jaegerstr. 17/19, D-52066 Aachen, Germany, e-mail: axel.mayer@rwth-aachen.de

This work was supported by the Deutsche Forschungsgemeinschaft (DFG; Grants No. KE 1664/1-2). 


\begin{abstract}
In this article, we present an approach for comprehensive analysis of the effectiveness of interventions based on nonlinear structural equation mixture models (NSEMM). We provide definitions of average and conditional effects and show how they can be computed. We extend the traditional moderated regression approach to include latent continous and discrete (mixture) variables as well as their higher-order interactions, quadratic or more general nonlinear relationships. This new approach can be considered a combination of the recently proposed EffectLiteR approach and the NSEMM approach. A key advantage of this synthesis is that it gives applied researchers the opportunity to gain greater insight into the effectiveness of the intervention. For example, it makes it possible to consider SEMs for situations where the treatment is non-effective for extreme values of a latent covariate but is effective for medium values, as we illustrate using an example from the educational sciences.
\end{abstract}

Keywords: Average and conditional effects, nonlinear structural equation mixture modeling, latent interactions, mixture modeling, non-normally distributed predictors. 


\section{Effect Analysis Using Nonlinear Structural Equation Mixture Modeling}

Evaluating the effectiveness of a treatment or an intervention is a relevant topic in empirical research. The main goal of this paper is to introduce a modern structural equation modeling approach that allows for comprehensive analysis of the effectiveness of interventions and treatments by including latent variables, quadratic and other nonlinear terms and mixtures. The new approach can be considered a combination of recent advancements in effect analysis (the EffectLiteR approach, Mayer, Dietzfelbinger, Rosseel, \& Steyer, 2016) and nonlinear structural equation mixture modeling (NSEMM, Kelava, Nagengast, \& Brandt, 2014). For example, we consider regressions such as the following

$$
E(\eta \mid X, \xi)=\gamma_{00}+\gamma_{01} \xi+\gamma_{02} \xi^{2}+\gamma_{10} X+\gamma_{11} X \xi+\gamma_{12} X \xi^{2}
$$

where $\eta$ and $\xi$ are latent variables measured by multiple indicators and $X$ is a binary treatment with values 0 and 1 . In contrast to standard moderated regression models, such a parameterization for the regression $E(\eta \mid X, \xi)$ makes it possible to consider situations where the treatment is non-effective for extreme values of the latent covariate but is effective for medium values of the latent covariate or vice versa. We discuss the definition of average and conditional effects in this particular model as well as in more general nonlinear models, their estimation as multigroup structural equation models, and the use of latent classes to account for unobserved heterogeneity and relax distributional assumptions.

The paper is structured as follows: We briefly introduce the standard moderated regression model with manifest variables and subsequently elaborate on complications that arise when nonlinear terms and/or latent variables are added to the model. We discuss out motivation for developing the new approach by highlighting its potential and the new options it opens up for expanded effect analysis. We first give an introduction to the EffectLiteR approach and the NSEMM approach separately before describing the combined model, along with some possible ways of estimating it. In the illustration section, we use an empirical example from the educational sciences to estimate average and conditional effects. Finally, we discuss the 
opportunities and limitations of the approach.

\section{Average Effects in a Manifest Moderated Regression Model}

The effects of an intervention or a treatment $X$ on a manifest dependent variable $Y$ taking into account a manifest continuous covariate $Z$ are usually modeled using a moderated regression model (Aiken \& West, 1991; Cohen, Cohen, West, \& Aiken, 2003; Preacher \& Hayes, 2004) in the form

$$
E(Y \mid X, Z)=\gamma_{00}+\gamma_{01} Z+\gamma_{10} X+\gamma_{11} X Z
$$

The average effect $\mathrm{AE}_{10}$ of treatment condition $X=1$ vs. $X=0$ is defined as the average difference between treatment groups, i.e., $\mathrm{AE}_{10}=E[E(Y \mid X=1, Z)-E(Y \mid X=0, Z)]$ and can be computed based on regression coefficients and the unconditional expectation of $Z$ as follows: $\mathrm{AE}_{10}=\gamma_{10}+\gamma_{11} E(Z)$ (e.g., Mayer et al., 2016). An average effect is sometimes also referred to as an average marginal effect (Greene, 2007; Williams, 2012).

A more common way to obtain the average effect is to mean center the continuous covariate $Z$, $\bar{Z}=Z-E(Z)$ and consider the regression:

$$
E(Y \mid X, \bar{Z})=\gamma_{00}^{*}+\gamma_{01}^{*} \bar{Z}+\gamma_{10}^{*} X+\gamma_{11}^{*} X \bar{Z} .
$$

The average effect then corresponds to $\mathrm{AE}_{10}=\gamma_{10}^{*}$. In linear models, both strategies give the exact same average effect but potentially different standard errors, because in the effect computation approach, we can account for the uncertainty that comes with estimating $E(Z)$. In the mean centering approach on the other hand, we subtract a fixed value, the sample estimate of $E(Z)$, prior to the analysis, so there is no way of accounting for its uncertainty. In the effect computation approach, we can take the joint distribution of the sample estimate of $E(Z)$ and the regression coefficients and use this information to compute point estimates and standard errors for the average effect which allows uncertainty to be taken into account (see Mayer et al., 2016 for 
more details). Note that the mean centering approach and the effect computation approach are identical if the true value of $E(Z)$ is known.

Oftentimes, we are not only interested in the average effect but also in conditional treatment effects for given values of the covariate(s). The differential effectiveness is represented by the regression coefficient $\gamma_{11}$ (or equivalently $\gamma_{11}^{*}$ ) of the interaction term. For a concrete value $z$ of $Z$, the conditional treatment effect is $\mathrm{CE}_{10}(z)=\gamma_{10}+\gamma_{11} z$, or when a value $\bar{z}$ is used, $\mathrm{CE}_{10}(\bar{z})=\gamma_{10}^{*}+\gamma_{11}^{*} \bar{z}$

While the moderated regression model is straightforward in models such as Equation 2, it is not as straightforward to extend it to models with conditional nonlinearities and latent variables. In the following sections, we elaborate on the problems that arise and provide a general approach to dealing with these problems.

\section{Quadratic and Other Nonlinear Terms}

An implicit assumption of the classic moderated regression approach that is oftentimes overlooked and rarely tested is that the regression above assumes a linear relationship between the outcome $Y$ and the covariate $Z$ in the control group and in the treatment group. However, if the true relationship between $Y$ and $Z$ in at least one of the treatment groups is actually nonlinear, we may erroneously conclude based on the conditional linearity model that there are no differential treatment effects, even though there are in fact differences in treatment effects (Ganzach, 1997; Lubinski \& Humphreys, 1990). In our empirical example, we illustrate such a case with real data. If we fit the conditional linearity model above, we will find a non-significant interaction term. But as soon as we allow for a potentially different quadratic relationship between $Y$ and $Z$ in both treatment groups, i.e., fit the model

$$
E(Y \mid X, Z)=\gamma_{00}+\gamma_{01} Z+\gamma_{02} Z^{2}+\gamma_{10} X+\gamma_{11} X Z+\gamma_{12} X Z^{2}
$$

we see that there are differential treatment effects. The differential treatment effects in this concrete example imply that people with medium values of the covariate benefit most from the 
treatment, whereas people with extreme values of the covariate (high or low) benefit less. This finding clearly shows the need for a more complex effect analysis that can incorporate nonlinear relationships between variables.

While mean centering is easy to use to compute average effects based on the standard moderated regression framework, it is no longer as easy when quadratic or other nonlinear terms come into play, because the interpretation of $\gamma_{10}^{*}$ changes. Note that the definition of an average effect introduced above, $\mathrm{AE}_{10}=E[E(Y \mid X=1, Z)-E(Y \mid X=0, Z)]$, does not depend on the concrete parameterization used for the regression $E(Y \mid X, Z)$. We can therefore also apply it to the quadratic model and compute the average effect based on Equation 3 as $\mathrm{AE}_{10}=\gamma_{10}+\gamma_{11} E(Z)+\gamma_{12} E\left(Z^{2}\right)$.

However, in nonlinear models such as quadratic models, the strategy of mean centering the covariate will no longer yield the average effect, i.e., in the regression:

$$
E(Y \mid X, \bar{Z})=\gamma_{00}^{*}+\gamma_{01}^{*} \bar{Z}+\gamma_{02}^{*} \bar{Z}^{2}+\gamma_{10}^{*} X+\gamma_{11}^{*} X \bar{Z}+\gamma_{12}^{*} X \bar{Z}^{2}
$$

the coefficient $\gamma_{10}^{*}$ is not equal to the average effect as defined in this article. The reason is that the squared centered covariate no longer has mean zero (e.g., Busemeyer \& Jones, 1983).

Sometimes double mean centering is recommended (e.g., Lin, Wen, Marsh, \& Lin, 2010). In double mean centering the quadratic term is first computed based on the centered covariate, and then the resulting quadratic term is centered again. The regression equation includes the double-centered quadratic term, and in this equation, $\gamma_{10}^{*}$ corresponds to the average effect. However, when using double mean centering, it is unclear what conditional expectation is modeled on the left-hand side of the equation, the interpretation of other coefficients may be obscured, and we can no longer easily compute conditional effects. In addition, double mean centering can not be generalized to more complex forms of nonlinear relationships. 


\section{Latent Variables and Multigroup SEM}

Another problem with the classic moderated regression framework is that including latent variables in the effect analysis is not straightforward. In the social and behavioral sciences situations where we cannot directly observe the values of some variables in the model are very common. Such latent variables further complicate the analysis of treatment effects in models with interactions and/or nonlinear terms. In manifest models, we can easily mean center variables and compute product terms of continuous and/or categorical variables before running the analysis. In latent variable models, we need advanced modeling techniques (e.g., Jedidi, Jagpal, \& DeSarbo, 1997; Kelava et al., 2014; Klein \& Moosbrugger, 2000; Klein \& Muthén, 2007; B. O. Muthén, 2001) to handle things like latent interactions or latent quadratic terms. A latent variable model could include several product terms consisting of all combinations of latent and/or manifest continuous as well as categorical variables. We distinguish four scenarios for products of different types of variables with different consequences for modeling:

(a) Product of a continuous latent variable and a continuous (latent) variable. If at least one of the variables involved in the product term is a continuous latent variable and the other one is a continuous (latent) variable, there exist a plethora of approaches that can be broadly classified into distribution analytic approaches (e.g., Kelava et al., 2011; Klein \& Moosbrugger, 2000), product indicator approaches (e.g., Jöreskog \& Yang, 1996; Kenny \& Judd, 1984; Marsh, Wen, \& Hau, 2004), Bayesian approaches (e.g., Lee, Song, \& Tang, 2007) and approaches based on factor scores (e.g., Wall \& Amemiya, 2003). Each of these approaches offers a different solutions to the problem that we cannot just compute the product term when latent variables are involved, because the values of the latent variables cannot be observed directly. Some of these approaches assume normal distributions for the variables that constitute the product, which is a strong assumption (e.g., Kelava \& Brandt, 2014).

(b) Product of a continuous latent variable and a categorical manifest variable. For 
scenarios in which one variable is a categorical manifest variable and the other is a continuous latent variable, the product term of the two variables can be modeled indirectly using a multigroup structural equation model (e.g., Bollen, 1989). As a simple example similar to Equation (2) but with a latent outcome and latent covariate, consider the regression $E(\eta \mid X, \xi)=\gamma_{00}+\gamma_{01} \xi+\gamma_{10} X+\gamma_{11} X \xi$. To indirectly model the product term $X \xi$, the outcome is regressed on the covariate in both treatment groups $x=0,1$ :

$$
E(\eta \mid X=x, \xi)=\beta_{x 0}+\beta_{x 1} \xi
$$

where $\beta$ s denote group-specific regression coefficients. The coefficient of the product term $\gamma_{11}$ is then computed as the difference between the two group-specific slopes, i.e., $\gamma_{11}=\beta_{11}-\beta_{01}$.

(c) Product of a categorical latent variable and a continuous (latent) variable. Models with categorical latent variables are widely known as latent class models or mixture models. Latent class structural equation models are similar to multigroup models with the exception that group membership is unknown. A distinction can be made between so-called direct and indirect approaches. In a direct approach, the latent classes are considered to be distinct subgroups within a heterogeneous population with potentially class-specific measurement and a class-specific structural model (e.g., Dolan \& van der Maas, 1998; Titterington, Smith, \& Makov, 1985). The direct approach can be used to model unobserved heterogeneity in the effects of one variable on the other, and the product term for the latent categorical variable and the (latent) continuous variable can be computed as shown for the multigroup model. In an indirect approach, all parameters except for the variance and the expected value of the mixtures are constrained over all latent classes (e.g., Bauer, 2005; Pek, Sterba, Kok, \& Bauer, 2009; Pek, Losardo, \& Bauer, 2011). The underlying assumption for this approach are not distinct subgroups but that a latent variable of interest is itself non-normally distributed. With this approach, non-normality of the latent 
dependent and independent variables and therefore their indicators can be taken into account (McLachlan \& Peel, 2000).

(d) Product of a categorical latent variable and a categorical manifest variable. If the latent variable and the manifest variable included in the product term are both categorical variables, we need a combination of the multigroup approach and the latent class model. In such models, the categorical manifest variable is sometimes called a knownclass variable (L. K. Muthén \& Muthén, 1998-2010). The groups/classes in this model are formed by building all possible combinations of values of the latent class variable and the manifest categorical variable. This unfolding strategy can also be used to model interactions between two or more categorical variables (Mayer et al., 2016).

These four scenarios for products of different types of variables illustrate the challenges substantive researchers face when modeling interactions with latent variables in a moderated regression framework. As soon as at least one variable is not directly observed, we need to go beyond computing simple products of (manifest) variables to adequately model interactions. The scenarios also show the need for an integrative approach that combines the aforementioned ways of handling latent interactions.

\section{Statistical Motivation}

Dealing with two challenges of classic moderated regression models, namely the unclear definition and computation of effects when nonlinear terms are involved and the complexities of modeling nonlinear terms when latent variables are involved, requires a new approach for comprehensive analysis of the effects of interventions based on nonlinear structural equation mixture modeling. To illustrate the need for a new approach, consider again Equation 1:

$$
E(\eta \mid X, \xi)=\gamma_{00}+\gamma_{01} \xi+\gamma_{02} \xi^{2}+\gamma_{10} X+\gamma_{11} X \xi+\gamma_{12} X \xi^{2} \quad \text { (Eq. 1, repeated) }
$$


Even in such a seemingly simple regression example with only six coefficients, a relatively complex combination of previously mentioned methods is required to adequately model the regression with latent variables: For the quadratic term $\xi^{2}$, we need a method for the product of two latent variables (e.g., a distribution analytic approach), for the interaction terms $X \xi$ and $X \xi^{2}$, we need a multigroup approach because $X$ is a binary variable, and if we want to account for the non-normality of $\xi$, we also need a indirect latent class approach. Finally, since we cannot easily center $\xi$ and $\xi^{2}$ in the two groups, we need clear definitions of average and conditional effects as well as the necessary computations to estimate these effects.

In this article, we therefore introduce a combination of the recently proposed EffectLiteR approach (Mayer et al., 2016; Mayer, Nagengast, Fletcher, \& Steyer, 2014) for analyzing average and conditional effects and nonlinear structural equation mixture modeling (NSEMM, Kelava et al., 2014) to provide applied researchers with modern technology to evaluate the effectiveness of an intervention or a treatment. The new approach applies to models such as Equation 1 but also other forms of interactions and nonlinearities. It goes beyond existing approaches in allowing for higher-order interactions between different types of variables, interactions involving nonlinear terms, and computations of effects in latent variable models.

\section{Substantive Motivation}

From a substantive point of view, nonlinear effects can be very helpful in examining treatment effects. Applied researchers develop interventions with the objective of helping those in need, e.g., fostering motivation in students with low motivation, or reducing anxiety among those individuals who suffer from intense anxiety. However, whether interventions are actually able to improve outcomes in extreme groups (i.e., individuals with especially high or low values in the variable of interest) is seldom assessed. There is thus a need to analyze conditional effects in detail.

In our empirical example from educational science, we will examine the relationship between self-efficacy and boredom in a control group and a treatment group. Previous findings suggest that students with higher ability report a higher level of boredom in school than their peers with lower 
ability (Plucker \& McIntyre, 1996). In addition, there have been controversial discussions on the association between students' required level of challenge in a lesson and resulting boredom. More specifically, Csikszentmihalyi (1975) argued that boredom results from a lack of challenge. By contrast, Götz, Frenzel, and Haag (2006) showed that students can face boredom both due to underchallenge (e.g., already knowing the content well) and overchallenge (e.g., problems with understanding). Research on the association between students' abilities and boredom (also regarding the comparison of high ability students with those of average or low ability) has yielded inconsistent results: positive, negative, curvilinear, or no associations have all been reported (for an overview see Farmer \& Sundberg, 1986; Preckel, Götz, \& Frenzel, 2010). In this paper, we test whether a u-shaped relationship between students' self-efficacy and boredom is influenced by a treatment (autonomy support versus no autonomy support). Autonomy support is assumed to affect students' boredom in class (Daschmann, Goetz, \& Stupnisky, 2011; Tze, Klassen, \& Daniels, 2014). To account for the expected complexity of the associations between self-efficacy and boredom, we explore whether students with moderate self-efficacy will show stronger effects than students with more extreme (low or high) values of self-efficacy. Both self-efficacy and boredom are measured by multiple indicators and are modeled as latent variables. Such a comprehensive effect analysis with latent variables would not have been possible with existing approaches.

\section{The EffectLiteR Approach}

We first introduce the EffectLiteR and NSEMM approaches separately, then we develop the combined model and apply it to our substantive example. The EffectLiteR approach is a comprehensive framework for evaluating the differential effectiveness of an intervention or a treatment. We denote the discrete treatment variable as $X$ with values $x=0,1, \ldots, p$, the continuous (latent) outcome variable as $\eta$, a single categorical covariate as $K$ with values $k=0,1, \ldots, j$, and a vector of (latent) covariates as $\boldsymbol{\xi}=\left(1, \xi_{1}, \xi_{2}, \ldots, \xi_{q}\right)^{\prime}$ using $z=0,1, \ldots, q$ to refer to entries in $\boldsymbol{\xi}$. When considering multiple categorical covariates, $K$ is obtained by unfolding 
all discrete covariates $K_{1}, K_{2}, \ldots$, i.e., the values of $K$ then represent all possible combinations of values of multiple categorical covariates. The regression of $\eta$ on $X, K$, and $\xi$ is denoted by $E(\eta \mid X, K, \xi)$, where different parameterizations for this regression can be considered.

\section{Nonparametric Definitions of Effects}

A core component of the EffectLiteR approach is the nonparametric definitions of average and conditional effects based on the regression $E(\eta \mid X, K, \boldsymbol{\xi})$. Under the assumption of no omitted confounders, these effects correspond to effect definitions given in the causal inference literature (e.g., Imbens \& Rubin, 2015; Rubin, 1974; Pearl, 2009; Steyer, Mayer, \& Fiege, 2014). In short, we define a conditional effect function whose values are the conditional effects of intervention $X=t, t=1,2, \ldots, p$, compared to the control group $X=0$ :

$$
\mathrm{CE}_{t 0}(K, \boldsymbol{\xi})=E(\eta \mid X=t, K, \boldsymbol{\xi})-E(\eta \mid X=0, K, \boldsymbol{\xi})
$$

Note that Mayer et al. (2016) used slightly different notation, first introducing the regression $E(\eta \mid X, K, \xi)$, which can always be written as:

$$
E(\eta \mid X, K, \boldsymbol{\xi})=g_{0}(K, \boldsymbol{\xi})+g_{1}(K, \boldsymbol{\xi}) \cdot I_{X=1}+\ldots+g_{p}(K, \boldsymbol{\xi}) \cdot I_{X=p}
$$

The $g_{t}(K, \boldsymbol{\xi})$ function in Equation 6 corresponds to the $\mathrm{CE}_{t 0}(K, \boldsymbol{\xi})$ function in Equation 5. Average and conditional effects are then unambiguously defined as expectations or conditional expectations of the effect function $\mathrm{CE}_{t 0}(K, \boldsymbol{\xi})$ (see also Table 1 in Mayer et al., 2016). For example, we may want to consider average effects, $E\left[\mathrm{CE}_{t 0}(K, \boldsymbol{\xi})\right]$, effects given a treatment condition $E\left[\mathrm{CE}_{t 0}(K, \boldsymbol{\xi}) \mid X=x\right]$, effects given values of one or more categorical covariates $E\left[\mathrm{CE}_{t 0}(K, \boldsymbol{\xi}) \mid K=k\right]$, effects given values of one or more continuous covariates $E\left[\mathrm{CE}_{t 0}(K, \boldsymbol{\xi}) \mid \boldsymbol{\xi}=\boldsymbol{\xi}\right]$ or combinations thereof. 


\section{Multigroup SEM with Stochastic Group Sizes}

To estimate average and conditional effects, we oftentimes need to specify a functional form for the regression $E(\eta \mid X, K, \boldsymbol{\xi})$ and a statistical model. In principle, we could, for example, use the general linear model, generalized linear models, or mixed models, among others. Mayer et al. (2016) propose computing the effects based on a multigroup structural equation model (multigroup SEM) with stochastic group sizes. This general approach has several advantages: It can easily deal with latent dependent variables and covariates, it allows for stochastic regressors, it naturally includes higher-order interactions, and many recent developments from the SEM literature can be incorporated into the effect analysis.

The complete EffectLiteR model consists of a group-invariant measurement model relating manifest variables in the vector $\mathbf{y}$ to latent variables in the vector $\boldsymbol{\eta}=\left(\eta, \xi_{1}, \xi_{2}, \ldots, \xi_{q}\right)^{\prime}$, a group-specific structural model specifying the regression of $\eta$ on $\boldsymbol{\xi}$, and a specification of the group sizes:

Group-invariant measurement model

$\mathbf{y}=v+\Lambda \eta+\varepsilon$

Structural model for group $(X=x, K=k)$

$$
\begin{aligned}
\boldsymbol{\eta} & =\boldsymbol{\alpha}_{x k}+\mathbf{B}_{x k} \boldsymbol{\eta}+\boldsymbol{\zeta} \\
& =\left(\begin{array}{c}
\alpha_{x k 0} \\
\mu_{x k 1} \\
\mu_{x k 2} \\
\vdots \\
\mu_{x k q}
\end{array}\right)+\left(\begin{array}{ccccc}
0 & \alpha_{x k 1} & \alpha_{x k 2} & \ldots & \alpha_{x k q} \\
0 & 0 & 0 & \ldots & 0 \\
0 & 0 & 0 & \ldots & 0 \\
\vdots & \vdots & \vdots & \ddots & \vdots \\
0 & 0 & 0 & \ldots & 0
\end{array}\right)\left(\begin{array}{c}
\eta \\
\xi_{1} \\
\xi_{2} \\
\vdots \\
\xi_{q}
\end{array}\right)+\left(\begin{array}{c}
\zeta_{0} \\
\zeta_{1} \\
\zeta_{2} \\
\vdots \\
\zeta_{q}
\end{array}\right)
\end{aligned}
$$

Group sizes for group $(X=x, K=k)$

$$
P(X=x, K=k)=f\left(\kappa_{x k}\right),
$$


where $v$ is a vector of group-invariant measurement intercepts, $\boldsymbol{\Lambda}$ is a matrix of group-invariant loadings, $\boldsymbol{\varepsilon}$ is a vector of measurement error variables, $\alpha_{x k z}$ are regression coefficients, $\mu_{x k z}=E\left(\xi_{z} \mid X=x, K=k\right), \zeta=\left(\zeta_{0}, \zeta_{1}, \ldots, \zeta_{q}\right)^{\prime}$ is a vector of structural residuals, and $\kappa_{x k}$ denote the parameters for the group sizes. Different functions can be chosen for $f$, depending on the exact parameterization of the model for the group sizes. While Mayer et al. (2016) suggested using a Poisson model, a multinomial logit model or a Bayesian model, as will be used later in this paper, are also possible, among others.

\section{Computing Effects}

Based on the parameters of the EffectLiteR structural model (Equation 8), we can derive the parameters of the conditional effects function $\mathrm{CE}_{t 0}(K, \xi)$ (Equation 5). Note that the specification as multigroup SEM with stochastic group sizes implies a specific functional form for the conditional effect function, which includes higher-order interactions:

$$
\mathrm{CE}_{t 0}(K, \boldsymbol{\xi})=\gamma_{t \mathbf{0}}^{\prime} \xi+\gamma_{t \mathbf{1}}^{\prime} \xi I_{K=1}+\gamma_{t \mathbf{2}}^{\prime} \xi I_{K=2}+\ldots+\gamma_{t j}^{\prime} \xi I_{K=j}
$$

where $\gamma_{t k}=\left(\gamma_{t k 0}, \gamma_{t k 1}, \ldots, \gamma_{t k q}\right)^{\prime}$ is a vector of regression coefficients, and $I_{K=k}$ is an indicator variable for $(K=k)$. The coefficients $\gamma_{t k}$ of the effect function are computed based on the coefficients $\boldsymbol{\alpha}_{x k}$ of the structural model (Equation 8):

$$
\begin{aligned}
& \gamma_{t 0}=\alpha_{t 0}-\alpha_{00} \quad \text { for } k=0 \\
& \gamma_{t k}=\alpha_{t k}-\alpha_{t 0}-\alpha_{0 k}+\alpha_{00} \quad \text { for } k \neq 0 .
\end{aligned}
$$

For a derivation of these results, see Equations 17 to 18 in Mayer et al. (2016). Once we have the parameters for the effect functions, we can compute average and conditional effects, such as $E\left[\mathrm{CE}_{t 0}(K, \boldsymbol{\xi})\right], E\left[\mathrm{CE}_{t 0}(K, \boldsymbol{\xi}) \mid X=x\right]$, or $E\left[\mathrm{CE}_{t 0}(K, \boldsymbol{\xi}) \mid K=k\right]$, based on these parameters and conditional and unconditional expectations of covariates (in the linear case). Since these computations can be tedious in complex examples, the open source $\mathrm{R}$ package EffectLiteR is 
available to do these computations automatically.

\section{The NSEMM Approach}

\section{Introduction to NSEMM}

The EffectLiteR approach builds on traditional multigroup SEM and therefore relies on ( $x k$ )-conditional linearity of the regression of $\eta$ on $\boldsymbol{\xi}$. If the classic maximum-likelihood based variance-covariance matrix of parameter estimates is used in combination with the delta method to obtain standard errors of average and conditional effects, we also need to assume (xk)-conditional normality of latent variables. Both assumptions are likely to be violated in models where the true relationship between $\eta$ and $\xi$ is nonlinear.

To deal with such scenarios, Kelava et al. (2014) developed the nonlinear structural equation mixture modeling approach (NSEMM approach), which combines the strength of a contemporary parametric nonlinear SEM (Klein \& Moosbrugger, 2000; Klein \& Muthén, 2007) and semiparametric structural equation mixture modeling (Arminger, Stein, \& Wittenberg, 1999; Bauer, 2005; Jedidi et al., 1997; B. O. Muthén, 2001).

\section{NSEMM Model}

The NSEMM model consists of a measurement model and a structural model. Both of them can potentially be latent class-specific, as in traditional structural equation mixture modeling (SEMM). In addition to classic SEMM, the NSEMM approach adds nonlinear terms for the latent predictors $\boldsymbol{\xi}$ in the structural model, so that the resulting model equations are

Measurement model for latent class $c$

$$
\mathbf{y}=\boldsymbol{v}_{c}+\boldsymbol{\Lambda}_{c} \boldsymbol{\eta}+\boldsymbol{\varepsilon}
$$

Structural model for latent class $c$

$$
\boldsymbol{\eta}=\boldsymbol{\alpha}_{c}+\mathbf{B}_{c} \boldsymbol{\eta}+\boldsymbol{\Gamma}_{c} h(\boldsymbol{\xi})+\boldsymbol{\zeta},
$$


where $\boldsymbol{\eta}=\left(\eta, \boldsymbol{\xi}^{\prime}\right)^{\prime}, h(\cdot)$ is a function that maps the vector $\boldsymbol{\xi}$ to a vector of product terms of $\boldsymbol{\xi}$ (e.g., interactions or quadratic terms), and $\boldsymbol{\Gamma}_{c}$ is a matrix of the nonlinear regression coefficients of $h(\boldsymbol{\xi})$ on $\boldsymbol{\eta}$. Note that we use slightly different notation. Kelava et al. (2014) introduce the NSEMM approach using the different equations for the $\mathbf{x}$-side and the $\mathbf{y}$-side of the model. In contrast to Kelava et al. (2014), the specification chosen above allows for the visualization of the parameters for the class-specific mean structure of the latent predictors $\boldsymbol{\xi}$ in the equation for the structural model (in $\boldsymbol{\alpha}_{c}$ ). For reasons that become apparent later in the article, this feature will be especially convenient for the combination of EffectLiteR and NSEMM, where these parameters are needed for the computation of average and conditional effects.

The NSEMM approach is a latent class model. Different parameter constraints need to be introduced to the model depending on the intended interpretation of these classes. As in traditional SEMM, a distinction can be made between the above mentioned direct approaches, where latent classes are considered to be distinct subgroups within a heterogeneous population with class-specific nonlinear relationships, and indirect approaches, where latent classes are used as a tool to account for non-normally distributed (latent) variables.

\section{Combined EffectLiteR and NSEMM Model}

Combining EffectLiteR and NSEMM opens up possibilities in both worlds. In the proposed synthesis of the two approaches, we extend the EffectLiteR approach to models with conditional nonlinearities and non-normalities and show how the NSEMM approach can be used to compute various kinds of effects of interest. The EffectLiteR approach benefits from the addition of quadratic and other nonlinear effects, semi-parametric relationships within groups, unobserved heterogeneity in regression coefficients (direct mixture approach), and non-normality of latent predictor variables. The NSEMM approach, meanwhile, benefits from the addition of clear definitions of average and conditional effects, effect sizes, stochastic group sizes, and higher-order interactions involving categorical variables. 


\section{The Combined Model}

In order to combine the EffectLiteR and the NSEMM approaches, we need a combination of latent classes and observed groups (intervention groups and values of categorical covariates), i.e., the classes in the combined model are formed by all possible combinations of latent classes and observed groups in the model. We define a new variable $C^{*}$ that is similar to $K$ in the EffectLiteR model, but can be considered a more general version of $K$ also including latent classes. The values $c^{*}$ of $C^{*}$ represent all possible combinations of values of (multiple) categorical covariates and latent classes. The combined model equations then consist of three parts: (1) the class-invariant measurement model from the EffectLiteR approach, (2) the class-specific structural model from the NSEMM approach possibly including quadratic and other nonlinear terms, and (3) the model for the stochastic class sizes from the EffectLiteR approach. Formally, the combined model equations are given by:

Invariant measurement model

$$
\mathbf{y}=v+\Lambda \eta+\varepsilon
$$

Structural model

$$
\begin{gathered}
\boldsymbol{\eta}=\boldsymbol{\alpha}_{x c^{*}}+\mathbf{B}_{x c^{*}} \boldsymbol{\eta}+\boldsymbol{\Gamma}_{x c^{*}} h(\boldsymbol{\xi})+\boldsymbol{\zeta} \\
\text { Model for group sizes } \\
P\left(X=x, C^{*}=c^{*}\right)=f\left(\kappa_{x c^{*}}\right) .
\end{gathered}
$$

Notice that we assume a group- and class-invariant measurement model for our effect model. The model for group sizes is not further specified. Depending on the method of estimation, different models are possible, including multinomial models, Poisson models, or Bayesian approaches.

\section{Computing Effects in the Combined Model}

Based on the parameters of the combined EffectLiteR-NSEMM model, we can again compute average and conditional effects of intervention $X=t$ vs. the control group $X=0$. A conditional 
effect given a value $c$ of $C^{*}$ and a value $\xi$ of $\xi$ is defined as:

$$
\mathrm{CE}_{t 0}\left(C^{*}=c^{*}, \boldsymbol{\xi}=\boldsymbol{\xi}\right)=E\left(\eta \mid X=t, C^{*}=c^{*}, \boldsymbol{\xi}=\boldsymbol{\xi}\right)-E\left(\eta \mid X=0, C^{*}=c^{*}, \boldsymbol{\xi}=\boldsymbol{\xi}\right)
$$

and can be computed by inserting the corresponding values into the structural model. The parametric form of the effect function itself, $\mathrm{CE}_{t 0}\left(C^{*}, \boldsymbol{\xi}\right)$, depends on the specification of the $h(\boldsymbol{\xi})$ function. The computation of the gamma coefficients (see Equations 10 and 11) generalizes to the combined EffectLiteR-NSEMM model, because the class-specific conditional regressions are linear in $(\xi, h(\xi))$. In our empirical example, we demonstrate the computations for the special case of quadratic regression in both intervention groups. Finally, we can compute average and various conditional effects as expectations and conditional expectations of $\mathrm{CE}_{t 0}\left(C^{*}, \boldsymbol{\xi}\right)$. In nonlinear models with latent classes, there is no general solution and the computation needs to be worked out for special cases, as we will illustrate for our quadratic model with a non-normal predictor.

\section{Estimation and Software}

The combined EffectLiteR and NSEMM model can be estimated in state-of-the-art software packages. Mplus (L. K. Muthén \& Muthén, 1998-2012) provides the knownclass/latent class option in combination with the LMS approach for latent interactions. Bayesian estimation is possible as well: In this article we used R (R Core Team, 2013) and rely on a slightly modified input generated by the R package blavaan (Merkle \& Rosseel, 2015), which itself provides an interface between lavaan (Rosseel, 2012) and JAGS (Plummer, 2015). Software code is provided in Web Appendices A to C. The R package nlsem (Umbach, Naumann, Brandt, \& Kelava, in press) can be used for some special cases of the model, but does not yet have an option for knownclasses as of writing this article. Other software packages may provide similar options but probably require additional effort by the user to specify the likelihood or the Bayesian model. 


\section{Illustrative Example}

\section{Research Question}

To illustrate the proposed EffectLiteR-NSEMM approach, we use an empirical example from a quasi-experimental field study in 16 ninth grade physics classrooms with one experimental group and one control group. In this example, we want to examine how effective the treatment (manipulated autonomy support by the teacher) is in reducing students' state of boredom and whether that treatment effect depends on students' self-efficacy. We modeled the relationship between boredom and self-efficacy in each treatment group as quadratic, because we wanted to allow for a potentially u-shaped relationship between boredom and self-efficacy: Students with very low or very high self-efficacy may be more bored compared to students with medium values of self-efficacy. A different quadratic relationship within treatment groups would then imply a $\mathrm{u}$-shaped form of the effect function. We hypothesize a u-shaped effect function, where the treatment is most effective in reducing boredom for medium values of self-efficacy and less effective for extreme values (high or low) of self-efficacy.

We do not present a comprehensive analysis of the study. Instead, the primary goal of our paper is to illustrate the EffectLiteR-NSEMM approach as a means of estimating average and conditional effects based on nonlinear structural equation mixture modeling. We restrict ourselves to boredom as the dependent variable and to only one latent predictor, self-efficacy. For an extensive analysis of the differential effects, see Flunger, Mayer, and Umbach (in prep.).

\section{Participants and Procedure}

We used a complete data set based on listwise deletion for illustrative purposes. The total sample size for which complete data is availabe is $N=297$, where $N_{c}=156$ students in 8 classes were assigned to the control group and $N_{t}=141$ (also 8 classes) were assigned to the treatment group. 29 students have been excluded due to missing values on one or more of the measures.

Data were gathered using student questionnaires at two time points in the second half of 2011 in physics classes in German academic track schools. First, trait measures (e.g., students' 
self-efficacy) were gathered. Approximately 2 months after the first point of data collection, state measures (e.g., boredom) were assessed while the regular physics teachers either taught a standardized autonomy-supportive teaching unit (experimental condition) or a standardized teaching unit that did not deliver autonomy support (control condition). The state measures were collected at three time points, once before the start of the lesson, once during the teaching unit (after around 45 minutes) and once at the end of the lesson. Both teaching units dealt with the principles of heat transfer (Denecke, Kirsch, \& Schuett, 2007; Duit \& Mikelskis-Seifert, 2010). This topic was chosen because it enabled us to implement an experiment in the teaching unit that could either be done by the students themselves (experimental condition) or be shown to the students (control condition).

To control for potential effects of students' pre-knowledge, classes in which students already had been taught about heat transfer and classes in which heat transfer had not been taught yet were randomly distributed in equal numbers to the experimental and control groups. In this example, we focus on the second state measurement of boredom, which was collected directly after students had carried out experiments on their own.

\section{Measures}

Boredom. In our analysis, we used the latent variable boredom as the dependent variable $\eta$. It was measured by a scale with two indicators (Cronbach's $\alpha=.80$ ) which were taken from Pekrun, Goetz, Zirngibl, v. Hofe, and Blum (2002):

$Y_{12}:$ I am bored.

$Y_{22}$ : I am so bored, I can barely keep myself awake.

The response format was a five-point Likert scale which ranged from 1 (not at all true) to 5 (completely true).

Treatment Variable. The autonomy-supportive intervention $X$ encompassed both a training for the physics teacher and a standardized teaching unit for the whole class of students. The intervention took place in two subsequent physics lessons (90 minutes). The training consisted of 
extensive information on autonomy supportive strategies in an informational leaflet and an informational training session for each teacher individually. Based on materials for teaching the principles of heat transfer (Denecke et al., 2007), a teaching unit that focused on three autonomy-supportive strategies (i.e., provision of rationales, choices, and informational language) was developed. The physics teachers in the control condition did not receive training and the teaching unit for the control condition did not entail autonomy-supportive strategies. In this condition, an experiment was shown to the students.

Self-efficacy. The scale 'self-efficacy' consisted of four items (Cronbach's $\alpha=.93$ ) adapted from Pekrun et al. (2002). We modeled a latent predictor $\xi$ with these four indicators:

$Y_{11}$ : In physics, I am sure that I can understand the most difficult material.

$Y_{21}$ : I am convinced that I can understand even the most complex material that the teacher introduces.

$Y_{31}$ : I am convinced that I can achieve good results in exams in physics.

$Y_{41}$ : I am convinced that I can master the skills taught in physics.

The response format was a five-point Likert scale which ranged from 1 (not at all true) to 5 (completely true).

\section{Statistical Model}

In our example, we want to illustrate two particular features of the EffectLiteR-NSEMM combination, namely quadratic effects and non-normal distributions of latent predictor variables in an effect analysis. So we fitted the following model: 
Group-invariant $\tau$-equivalent measurement model

$$
\left(\begin{array}{l}
Y_{12} \\
Y_{22} \\
Y_{11} \\
Y_{21} \\
Y_{31} \\
Y_{41}
\end{array}\right)=\left(\begin{array}{l}
0 \\
v_{1} \\
0 \\
v_{2} \\
v_{3} \\
v_{4}
\end{array}\right)+\left(\begin{array}{ll}
1 & 0 \\
1 & 0 \\
0 & 1 \\
0 & \lambda_{1} \\
0 & \lambda_{2} \\
0 & \lambda_{3}
\end{array}\right)\left(\begin{array}{l}
\eta \\
\xi
\end{array}\right)+\left(\begin{array}{l}
\varepsilon_{12} \\
\varepsilon_{22} \\
\varepsilon_{11} \\
\varepsilon_{21} \\
\varepsilon_{31} \\
\varepsilon_{41}
\end{array}\right)
$$

Group-specific structural model

$$
\left(\begin{array}{l}
\eta \\
\xi
\end{array}\right)=\left(\begin{array}{l}
\alpha_{x 0} \\
\mu_{c 1}
\end{array}\right)+\left(\begin{array}{cc}
0 & \alpha_{x 1} \\
0 & 0
\end{array}\right)\left(\begin{array}{l}
\eta \\
\xi
\end{array}\right)+\left(\begin{array}{c}
\alpha_{x 2} \\
0
\end{array}\right)\left(\xi^{2}\right)+\left(\begin{array}{l}
\zeta_{0} \\
\zeta_{1}
\end{array}\right)
$$

Group sizes

$$
P(C=c)=\kappa_{c}
$$

Note that we treat the group sizes of the treatment groups as fixed, because they have been fixed by the experimental design. We only model the size of the latent classes as stochastic. A path diagram of the model is shown in Figure 1.

\section{Effect Model}

The effect model is based on the regression:

$$
E(\eta \mid X, \xi)=\gamma_{00}+\gamma_{01} \xi+\gamma_{02} \xi^{2}+\gamma_{10} X+\gamma_{11} X \xi+\gamma_{12} X \xi^{2}
$$

where the regression coefficients can be computed based on model parameters with $\gamma_{00}=\alpha_{00}$, $\gamma_{01}=\alpha_{01}, \gamma_{02}=\alpha_{02}, \gamma_{10}=\alpha_{10}-\alpha_{00}, \gamma_{11}=\alpha_{11}-\alpha_{01}$, and $\gamma_{12}=\alpha_{12}-\alpha_{02}$. Because the chosen functional form of both treatment-group specific regressions is quadratic, the effect function, 
which is their difference, is also quadratic:

$$
\begin{aligned}
\mathrm{CE}_{10}(\xi) & =E(\eta \mid X=1, \xi)-E(\eta \mid X=0, \xi) \\
& =\gamma_{10}+\gamma_{11} \xi+\gamma_{12} \xi^{2}
\end{aligned}
$$

To compute the average effect, we also need the unconditional expectations of $\xi$ and $\xi^{2}$. These can also be computed based on model parameters using standard formulas for conditional and unconditional expectations:

$$
\begin{aligned}
& E(\xi)=\alpha_{00} \cdot P(C=0)+\alpha_{10} \cdot P(C=1), \\
& E\left(\xi^{2}\right)=E\left(\xi^{2} \mid C=0\right) \cdot P(C=0)+E\left(\xi^{2} \mid C=1\right) \cdot P(C=1) .
\end{aligned}
$$

where $E\left(\xi^{2} \mid C=0\right)=\alpha_{00}^{2}+\operatorname{Var}\left(\zeta_{1} \mid C=0\right)$ and $E\left(\xi^{2} \mid C=1\right)=\alpha_{10}^{2}+\operatorname{Var}\left(\zeta_{1} \mid C=1\right)$. The average effect is $E\left[\mathrm{CE}_{10}(\xi)\right]=\gamma_{10}+\gamma_{11} E(\xi)+\gamma_{12} E\left(\xi^{2}\right)$, and various conditional effects can be computed by inserting values of $\xi$ into the effect function. For example, the conditional effect for $\xi=1$ is $\mathrm{CE}_{10}(\xi=1)=\gamma_{10}+\gamma_{11}+\gamma_{12}$

\section{Results}

\section{Results for the NSEMM Model}

The results from our NSEMM model are summarized in Table 1. The table shows all estimated model parameters for the basis model. Notice that the effects of interest are not parameters of the basis model, but will be computed subsequently based on the fitted model. In addition, we plot the two group-specific regressions in Figure 2. By visual inspection, we see a quadratic relationship in the treatment group, but not in the control group. This is also reflected in the parameter for the quadratic effect in the control group $\left(\alpha_{02}=-0.01, \mathrm{CI}=[-0.16,0.15]\right) \mathrm{vs}$. the parameter in the treatment group $\left(\alpha_{12}=0.36, \mathrm{CI}=[0.18,0.55]\right)$. 


\section{Results for the Effect Analysis}

Based on the model parameters (Table 1), we computed the parameters of the effect function, the unconditional expectations of $\xi$ and $\xi^{2}$, as well as several average and conditional effects of interest. The results of the effect analysis are shown in Table 2. The average effect of the treatment is $\left.E\left[\mathrm{CE}_{10}(\xi)\right]=-0.73, \mathrm{CI}=[-0.98,-0.47]\right)$, indicating an average effect of the autonomy-supportive intervention in reducing boredom in students. We also computed different conditional effects for values 1 to 4 of self-efficacy (see Table 2). To further illustrate the conditional effect function, we plotted the function in Figure 3, which is the difference between the two group-specific regressions shown in Figure 2. It illustrates that the treatment is most effective in reducing boredom for medium values of self-efficacy and less effective for extreme values of self-efficacy. It can be seen that for extreme values of self-efficacy, the $95 \%$ credibility intervals of the conditional effects of the autonomy-supportive intervention do contain zero, whereas for medium values of self-efficacy, the credibility intervals no longer contain zero.

Notice that due to the nonlinear relationship, the average effect is not identical to the effect at the mean of the covariate: Compare the average effect (Table 2) with the value of the effect function at the mean of $\xi$, which is $E(\xi)=2.84$, in the effect plot (Figure 3). The two are different, and which one is more relevant depends on what we are interested in: The average effect gives the population level effect, and the effect at $\xi=2.84$ gives the conditional effect for a person with this particular value of $\xi$.

Another interesting aspect of our empirical example is that when we use the standard specification for a moderated regression model without the quadratic term, the coefficient of the interaction term is very close to zero and its credibility interval includes zero $\left(\gamma_{11}=0.01, \mathrm{CI}=[-0.24,0.24]\right)$. So, based on the misspecified model, we would erroneously conclude that the autonomy-supportive intervention is equally effective for everybody and we would miss an important aspect of the differential effectiveness. 


\section{Discussion}

In this article, we presented an approach for comprehensive analysis of the effectiveness of interventions based on nonlinear structural equation mixture modeling. The proposed approach offers new ways to evaluate the differential effectiveness of interventions for substantive researchers in experimental and quasi-experimental studies by expanding the standard modeling approach to treatment effects. It goes beyond the traditional moderated regression techniques by allowing for the incorporation of latent continuous and discrete variables, higher-order interactions of treatments and covariates, and conditionally nonlinear terms, which are useful for more differentiated modeling of covariate and outcome relationships. The proposed approach also extends more recent models for analyzing moderation effects. For example, it adds latent nonlinear terms and the mixture components for non-normally distributed predictors to the EffectLiteR model, it adds effect definitions and computations to the NSEMM model, and it adds stochastic regressors and stochastic group sizes to the multigroup structural equation modeling approach. The proposed extensions can help researchers gain new insights into the differential effectiveness of interventions, as we illustrated in our empirical example. With traditional approaches, it would have been impossible to consider the interaction between the categorical treatment variable and the squared latent covariate as well as the non-normal distribution of the predictor. With traditional approaches, it would also have been unclear how to obtain average and conditional effects on the basis of such a model.

\section{Advantages of the Proposed Approach}

Given the need to examine treatment effects in more detail, several issues have to be considered that go along with the specification and estimation of nonlinear regression models with latent variables. It is here that the advantages of the proposed approach come into play.

First, it is clear that nonlinear effects can be helpful in examining treatment effects. In our empirical example from educational science, we examined the relationship between self-efficacy and boredom. We tested whether a u-shaped (i.e., quadratic) relationship between self-efficacy 
and boredom is influenced by a treatment. This means that students with moderate self-efficacy showed stronger effects than students with more extreme (low or high) values of self-efficacy. The examination of the differential treatment effect is only made possible by the inclusion of higher-order interactions of treatments and latent covariates. However, choosing the appropriate type of nonlinearity (for example, u-shaped or logistic curves) is an issue which needs to be addressed separately. In some situations, it could make sense to apply even more flexible types of functions (e.g., regression splines) in order to approximate an unknown nonlinear relationship between latent covariates and outcome.

Second, integrating covariates into regression models when treatment effects are examined does not prevent unobserved heterogeneity of the treatment effects. Omitting covariates could lead to varying relationships and (conditional and unconditional) treatment effects across unobserved groups. Therefore, it makes sense to test for unobserved heterogeneity by applying a mixture modeling approach (e.g., McLachlan \& Peel, 2000). For example, it could happen that a specific (unknown) group of students does not respond to a treatment/intervention because they did not pay attention during an autonomy training. Or students might differ strongly in their (unknown) motivation to participate in an autonomy supportive learning setting. That is, if students believe that their own ability in a subject is low, an intervention might have negative effects for them (e.g., Durik, Shechter, Noh, Rozek, \& Harackiewicz, 2015), because even though they might be allowed to choose amongst different tasks, they might have the feeling that they are not able to solve any of them successfully. In addition, if students have notably high competence beliefs in an area, they could also easily get demotivated through an autonomy supportive intervention which is aimed at the pace of regular students. Applying mixture modeling techniques as proposed in our approach offers many possibilities to detect such sub-groups, even if the driving variables have not been observed.

Third, the estimation of nonlinear (manifest and latent) models depends strongly on distributional assumptions. For example, most procedures for the estimation of latent nonlinear effects assume normality of the latent variables (e.g., Klein \& Moosbrugger, 2000; Marsh et al., 
2004). This assumption leads to biased estimates and increased Type I error rates (e.g., Brandt, Kelava, \& Klein, 2014). Therefore, in order to obtain correct inferences about (conditional and unconditional) treatment effects, it is important to account for a potential non-normal distribution of the latent variables. This can be done by applying a mixture distribution to approximate the non-normality of the latent variables. This was done in our empirical example.

\section{Limitations and Future Directions}

The proposed approach extends the possibilities for a differential evaluation of treatment effects in a latent variable framework. However, there are several aspects which are not covered by the proposed approach.

First, the proposed approach does not account for clustered (i.e., nested) data structures as they are typically encountered in many disciplines, including educational sciences. When dealing with clustered data, these dependencies should be controlled for in order to obtain unbiased estimates and standard errors of conditional and average treatment effects. In the same vein, random coefficients, which could account for the heterogeneity of relationships between latent covariates and outcomes, are not part of the proposed approach. The proposed approach could be extended to overcome this limitation. Furthermore, to the best of our knowledge, current statistical software does not offer ready-to-use frequentist estimators or implementations of such a model.

Second, the proposed approach concentrates on very specific parametric (nonlinear) relationships between latent variables. In recent years, several Bayesian approaches have been proposed that allow for very flexible semi-parametric relationships (e.g., regression splines; Kelava \& Brandt, 2014). This limitation could be overcome by broadening the proposed framework for such relationships. However, in this context, deriving conditional and average effects is not straightforward, because higher order central and non-central moments need to be incorporated (Brandt, Umbach, \& Kelava, 2015). Nevertheless, accounting for a situation in which latent covariates have a flexible relationship with the outcome is necessary for differential and more realistic modeling of treatment or intervention effects. 
Third, although the implementation of the proposed approach as presented in our empirical example is given in the appendix, there are no ad-hoc routines within popular statistical software packages which can be applied by substantive researchers. This group is very interested in knowing how treatments unfold within specific intervals of a latent covariate (for example, a substantive research question could be: How large is the intervention effect in the face of severe levels of depression?). Thus, easy to use implementations of the proposed approach could enhance its use across a broader audience. 


\section{References}

Aiken, L. S., \& West, S. G. (1991). Multiple regression: Testing and interpreting interactions. Newbury Park, CA: Sage.

Arminger, G., Stein, P., \& Wittenberg, J. (1999). Mixtures of conditional mean- and covariance-structure models. Psychometrika, 64(4), 475-494. doi: http://dx.doi.org/10.1007/BF02294568

Bauer, D. J. (2005). A semiparametric approach to modeling nonlinear relations among latent variables. Structural Equation Modeling, 12, 513-535.

Bollen, K. A. (1989). Structural equation modeling with latent variables. New York, NY: Wiley.

Brandt, H., Kelava, A., \& Klein, A. (2014). A simulation study comparing recent approaches for the estimation of nonlinear effects in SEM under the condition of nonnormality. Structural Equation Modeling, 21, 181-195. doi: http://dx.doi.org/10.1080/10705511.2014.882660

Brandt, H., Umbach, N., \& Kelava, A. (2015). The standardization of linear and nonlinear effects in direct and indirect applications of structural equation mixture models for normal and nonnormal data. Frontiers in psychology, 6. doi: http://dx.doi.org/10.3389/fpsyg.2015.01813

Busemeyer, J. R., \& Jones, L. E. (1983). Analysis of multiplicative combination rules when the causal variables are measured with error. Psychological Bulletin, 93(3), 549-562. doi: http://dx.doi.org/10.1037/0033-2909.93.3.549

Cohen, J., Cohen, P., West, S. G., \& Aiken, L. S. (2003). Applied multiple regression/correlation analysis for the behavioral sciences (3rd ed.). Mahwah, NJ: Lawrence Erlbaum.

Csikszentmihalyi, M. (1975). Play and intrinsic rewards. Journal of Humanistic Psychology.

Daschmann, E. C., Goetz, T., \& Stupnisky, R. H. (2011). Testing the predictors of boredom at school: Development and validation of the precursors to boredom scales. British Journal of Educational Psychology, 81, 421-440. doi: http://dx.doi.org/10.1348/000709910X526038

Denecke, I., Kirsch, T., \& Schuett, D. (2007). Bausteine zum Thema Wärmeenergie. Schulset Hamburg. [Modules on heat energy]. Hamburg: Freie und Hansestadt Hamburg, Behörde 
für Bildung und Sport, Amt für Bildung, and Kiel: Projekt Physik im Kontext, IPN Leibniz-Institut für die Pädagogik der Naturwissenschaften: Kiel.

Dolan, C. V., \& van der Maas, H. L. J. (1998). Fitting multivariate normal finite mixtures subject to structural equation modeling. Psychometrika, 63, 227-253.

Duit, R., \& Mikelskis-Seifert, S. (2010). Physik im kontext. Konzepte, Ideen, Materialien für effizienten Physikunterricht. (Seelze: Friedrich Verlag)

Durik, A. M., Shechter, O. G., Noh, M., Rozek, C. S., \& Harackiewicz, J. M. (2015). What if I can't? Success expectancies moderate the effects of utility value information on situational interest and performance. Motivation and Emotion, 39, 104-118. doi: http://dx.doi.org/10.1007/s11031-014-9419-0

Farmer, R., \& Sundberg, N. D. (1986). Boredom proneness - the development and correlates of a new scale. Journal of Personality Assessment, 50, 4-17. doi: http://dx.doi.org/10.1207/s15327752jpa5001_2

Flunger, B., Mayer, A., \& Umbach, N. (in prep.). Beneficial for some or for everyone? Student characteristics as moderators of an autonomy-supportive intervention in the classroom. (Manuscript in preparation)

Ganzach, Y. (1997). Misleading interaction and curvilinear terms. Psychological Methods, 2(3), 235-247. doi: http://dx.doi.org/10.1037/1082-989X.2.3.235

Götz, T., Frenzel, A. C., \& Haag, L. (2006). Ursachen von Langweile im Unterricht. Empirische Pädagogik, 20(2), 113-134.

Greene, W. (2007). Econometric analysis. Upper Saddle River, NJ: Prentice-Hall.

Imbens, G. W., \& Rubin, D. B. (2015). Causal inference in statistics, social, and biomedical sciences. Cambridge University Press. doi: http://dx.doi.org/10.1017/cbo9781139025751

Jedidi, K., Jagpal, H. S., \& DeSarbo, W. S. (1997). STEMM: A general finite mixture structural equation model. Journal of Classification, 14, 23-50. doi: http://dx.doi.org/10.1007/s003579900002

Jöreskog, K. G., \& Yang, F. (1996). Nonlinear structural equation models: The Kenny-Judd 
model with interaction effects. In G. Marcoulides \& R. Schumaker (Eds.), Advanced structural equation modling - Issues and techniques (pp. 57-81). Mahwah, NJ: Lawrence Erlbaum.

Kelava, A., \& Brandt, H. (2014). A general nonlinear multilevel structural equation mixture model. Frontiers in Psychology, 5(748). doi: http://dx.doi.org/10.3389/fpsyg.2014.00748

Kelava, A., Nagengast, B., \& Brandt, H. (2014). A nonlinear structural equation mixture modeling approach for nonnormally distributed latent predictor variables. Structural Equation Modeling, 21, 468-481. doi: http://dx.doi.org/10.1080/10705511.2014.915379

Kelava, A., Werner, C., Schermelleh-Engel, K., Moosbrugger, H., Zapf, D., Ma, Y., ... West, S. G. (2011). Advanced nonlinear structural equation modeling: Theoretical properties and empirical application of the distribution-analytic LMS and QML estimators. Structural Equation Modeling, 18, 465-491.

Kenny, D. A., \& Judd, C. M. (1984). Estimating the nonlinear and interactive effects of latent variables. Psychological Bulletin, 96, 201-210. doi: http://dx.doi.org/10.1037/0033-2909.96.1.201

Klein, A. G., \& Moosbrugger, H. (2000). Maximum likelihood estimation of latent interaction effects with the LMS method. Psychometrika, 65, 457-474. doi: http://dx.doi.org/10.1007/BF02296338

Klein, A. G., \& Muthén, B. O. (2007). Quasi-maximum likelihood estimation of structural equation models with multiple interaction and quadratic effects. Multivariate Behavioral Research, 42, 647-673. doi: http://dx.doi.org/10.1080/00273170701710205

Lee, S.-Y., Song, X.-Y., \& Tang, N.-S. (2007). Bayesian methods for analyzing structural equation models with covariates, interaction and quadratic latent variables. Structural Equation Modeling, 14, 404-434. doi: http://dx.doi.org/10.1080/10705510701301511

Lin, G.-C., Wen, Z., Marsh, H. W., \& Lin, H.-S. (2010). Structural equation models of latent interactions: Clarification of orthogonalizing and double-mean-centering strategies. Structural Equation Modeling, 17, 374-391. doi: 
http://dx.doi.org/10.1080/10705511.2010.488999

Lubinski, D., \& Humphreys, L. G. (1990). Assessing spurious "moderator effects": Illustrated substantively with the hypothesized ("synergistic") relation between spatial and mathematical ability. Psychological Bulletin, 107(3), 385-393. doi: http://dx.doi.org/10.1037/0033-2909.107.3.385

Marsh, H. W., Wen, Z., \& Hau, K. T. (2004). Structural equation models of latent interactions: Evaluation of alternative estimation strategies and indicator construction. Psychological Methods, 9, 275-300. doi: http://dx.doi.org/10.1037/1082-989X.9.3.275

Mayer, A., Dietzfelbinger, L., Rosseel, Y., \& Steyer, R. (2016). The EffectLiteR approach for analyzing average and conditional effects. Multivariate Behavioral Research, 51, 374-391. doi: http://dx.doi.org/10.1080/00273171.2016.1151334

Mayer, A., Nagengast, B., Fletcher, J., \& Steyer, R. (2014). Analyzing average and conditional effects with multigroup multilevel structural equation models. Frontiers in Psychology, 5. doi: http://dx.doi.org/10.3389/fpsyg.2014.00304

McLachlan, G. J., \& Peel, D. (2000). Finite mixture models. New York: John Wiley \& Sons. doi: http://dx.doi.org/10.1002/0471721182

Merkle, E. C., \& Rosseel, Y. (2015). blavaan: Bayesian structural equation models via parameter expansion. Retrieved from http://arxiv.org/abs/1511.05604

Muthén, B. O. (2001). Second-generation structural equation modeling with a combination of categorical and continuous latent variables: New opportunities for latent class / latent growth modeling. In L. Collins \& A. Sayer (Eds.), New methods for the analysis of change (pp. 291-322). Washington, DC: American Psychological Association. doi: http://dx.doi.org/10.1037/10409-010

Muthén, L. K., \& Muthén, B. O. (1998-2010). Mplus LANGUAGE ADDENDUM (6.1 ed.) [Computer software manual]. Los Angeles, CA: Muthén \& Muthén.

Muthén, L. K., \& Muthén, B. O. (1998-2012). Mplus User's Guide (7th ed.) [Computer software manual]. Los Angeles, CA: Muthén \& Muthén. 
Pearl, J. (2009). Causality: Models, reasoning, and inference (2nd ed.). Cambridge, UK. doi: http://dx.doi.org/10.1017/cbo9780511803161

Pek, J., Losardo, D., \& Bauer, D. J. (2011). Confidence intervals for a semiparametric approach to modeling nonlinear relations among latent variables. Structural Equation Modeling, 18, $537-553$.

Pek, J., Sterba, S. K., Kok, B. E., \& Bauer, D. J. (2009). Estimating and visualizing nonlinear relations among latent variables: A semiparametric approach. Multivariate Behavioral Research, 44, 407-436.

Pekrun, R., Goetz, S., Jullien, Zirngibl, A., v. Hofe, R., \& Blum, W. (2002). Skalenhandbuch PALMA: 1. Messzeitpunkt (5. Klassenstufe). . Universität München: Institut Pädagogische Psychologie.

Plucker, \& McIntyre. (1996). Academic survivability. Gifted Child Quartlerly, 40.

Plummer, M. (2015). JAGS Version 4.0.0 user manual [Computer software manual].

Preacher, K. J., \& Hayes, A. F. (2004). SPSS and SAS procedures for estimating indirect effects in simple mediation models. Behavior Research Methods, Instruments, $\mathcal{F}$ Computers, 36(4), 717-731. doi: http://dx.doi.org/10.3758/BF03206553

Preckel, F., Götz, T., \& Frenzel, A. (2010). Ability grouping of gifted students: Effects on academic self-concept and boredom. British Journal of Educational Psychology, 80, 451-472. doi: http://dx.doi.org/10.1348/000709909X480716

R Core Team. (2013). R: A language and environment for statistical computing [Computer software manual]. Vienna, Austria. Retrieved from http://www.R-project.org/

Rosseel, Y. (2012). lavaan: An R package for structural equation modeling. Journal of Statistical Software, 48(2), 1-36. doi: http://dx.doi.org/10.18637/jss.v048.i02

Rubin, D. B. (1974). Estimating causal effects of treatments in randomized and nonrandomized studies. Journal of Educational Psychology, 66, 688-701. doi: http://dx.doi.org/10.1037/h0037350

Steyer, R., Mayer, A., \& Fiege, C. (2014). Causal inference on total, direct, and indirect effects. 
In Michalos, A. C. (Ed.), Encyclopedia of Quality of Life and Well-Being Research (pp. 606-631). Dordrecht, Netherlands: Springer. doi: http://dx.doi.org/10.1007/978-94-007-0753-5_295

Titterington, D. M., Smith, A. F. M., \& Makov, U. E. (1985). Statistical analysis of finite mixture distributions. Chichester, England: Wiley.

Tze, V. M., Klassen, R. M., \& Daniels, L. M. (2014). Patterns of boredom and its relationship with perceived autonomy support and engagement. Contemporary Educational Psychology, 39, 175-187. doi: http://dx.doi.org/10.1016/j.cedpsych.2014.05.001

Umbach, N., Naumann, K., Brandt, H., \& Kelava, A. (in press). Fitting nonlinear structural equation models in R with package nlsem. Journal of Statistical Software.

Wall, M. M., \& Amemiya, Y. (2003). A method of moments technique for fitting interaction effects in structural equation models. British Journal of Mathematical and Statistical Psychology, 56, 47-63. doi: http://dx.doi.org/10.1348/000711003321645331

Williams, R. (2012). Using the margins command to estimate and interpret adjusted predictions and marginal effects. The Stata Journal, 12, 308-331. 
Table 1

Estimated Parameters of the basis NSEMM model.

\begin{tabular}{|c|c|c|c|c|c|}
\hline \multirow[b]{2}{*}{ Coefficient } & \multirow[b]{2}{*}{ Mean } & \multirow[b]{2}{*}{$\mathrm{SD}$} & \multicolumn{3}{|c|}{ Quantiles } \\
\hline & & & $2.5 \%$ & $50 \%$ & $97.5 \%$ \\
\hline$v_{1}$ & -0.77 & 0.05 & -0.88 & -0.77 & -0.66 \\
\hline$v_{2}$ & 0.12 & 0.11 & -0.11 & 0.12 & 0.33 \\
\hline$v_{3}$ & 0.79 & 0.13 & 0.52 & 0.79 & 1.05 \\
\hline$v_{4}$ & 1.01 & 0.13 & 0.74 & 1.01 & 1.26 \\
\hline$\lambda_{1}$ & 0.95 & 0.04 & 0.88 & 0.95 & 1.03 \\
\hline$\lambda_{2}$ & 0.88 & 0.04 & 0.79 & 0.88 & 0.97 \\
\hline$\lambda_{3}$ & 0.79 & 0.04 & 0.71 & 0.79 & 0.88 \\
\hline$\alpha_{00}$ & 3.87 & 0.60 & 2.73 & 3.85 & 5.07 \\
\hline$\alpha_{10}$ & 5.67 & 0.71 & 4.32 & 5.66 & 7.10 \\
\hline$\alpha_{01}$ & -0.16 & 0.46 & -1.09 & -0.15 & 0.68 \\
\hline$\alpha_{11}$ & -2.26 & 0.54 & -3.36 & -2.26 & -1.22 \\
\hline$\alpha_{02}$ & -0.01 & 0.08 & -0.16 & -0.01 & 0.15 \\
\hline$\alpha_{12}$ & 0.36 & 0.09 & 0.18 & 0.36 & 0.55 \\
\hline $\operatorname{Var}\left(\zeta_{0} \mid X=0\right)$ & 1.20 & 0.17 & 0.90 & 1.19 & 1.57 \\
\hline $\operatorname{Var}\left(\zeta_{0} \mid X=1\right)$ & 0.70 & 0.11 & 0.49 & 0.69 & 0.94 \\
\hline$\mu_{01}$ & 1.76 & 0.18 & 1.47 & 1.74 & 2.18 \\
\hline$\mu_{11}$ & 3.54 & 0.17 & 3.22 & 3.53 & 3.89 \\
\hline $\operatorname{Var}\left(\zeta_{1} \mid C=0\right)$ & 0.32 & 0.13 & 0.15 & 0.30 & 0.64 \\
\hline $\operatorname{Var}\left(\zeta_{1} \mid C=1\right)$ & 0.57 & 0.15 & 0.32 & 0.55 & 0.90 \\
\hline$P(C=0)$ & 0.39 & 0.09 & 0.22 & 0.38 & 0.60 \\
\hline$P(C=1)$ & 0.61 & 0.09 & 0.40 & 0.62 & 0.78 \\
\hline $\operatorname{Var}\left(\varepsilon_{12} \mid X=0\right)$ & 0.55 & 0.12 & 0.32 & 0.54 & 0.81 \\
\hline $\operatorname{Var}\left(\varepsilon_{12} \mid X=1\right)$ & 0.38 & 0.08 & 0.24 & 0.38 & 0.55 \\
\hline $\operatorname{Var}\left(\varepsilon_{22} \mid X=0\right)$ & 0.56 & 0.12 & 0.34 & 0.55 & 0.81 \\
\hline $\operatorname{Var}\left(\varepsilon_{22} \mid X=1\right)$ & 0.31 & 0.07 & 0.18 & 0.30 & 0.46 \\
\hline $\operatorname{Var}\left(\varepsilon_{11} \mid X=0\right)$ & 0.24 & 0.05 & 0.15 & 0.23 & 0.34 \\
\hline $\operatorname{Var}\left(\varepsilon_{11} \mid X=1\right)$ & 0.16 & 0.03 & 0.10 & 0.16 & 0.24 \\
\hline $\operatorname{Var}\left(\varepsilon_{21} \mid X=0\right)$ & 0.32 & 0.05 & 0.24 & 0.32 & 0.44 \\
\hline $\operatorname{Var}\left(\varepsilon_{21} \mid X=1\right)$ & 0.21 & 0.04 & 0.15 & 0.21 & 0.30 \\
\hline $\operatorname{Var}\left(\varepsilon_{31} \mid X=0\right)$ & 0.34 & 0.06 & 0.24 & 0.34 & 0.46 \\
\hline $\operatorname{Var}\left(\varepsilon_{31} \mid X=1\right)$ & 0.43 & 0.06 & 0.32 & 0.43 & 0.57 \\
\hline $\operatorname{Var}\left(\varepsilon_{41} \mid X=0\right)$ & 0.36 & 0.06 & 0.27 & 0.36 & 0.48 \\
\hline $\operatorname{Var}\left(\varepsilon_{41} \mid X=1\right)$ & 0.43 & 0.06 & 0.32 & 0.42 & 0.56 \\
\hline
\end{tabular}

Note. $X$ is the treatment variable, $C$ is the latent class variable, $v_{1}$ to $v_{4}$ are measurement intercepts, $\lambda_{1}$ to $\lambda_{3}$ are loadings, $\varepsilon_{12}$ to $\varepsilon_{41}$ are measurement error variables, $\alpha_{00}$ to $\alpha_{12}$ are regression coefficients for the structural model, $\zeta_{0}$ and $\zeta_{1}$ are structural residuals, $\mu_{01}$ and $\mu_{11}$ are group-specific means of $\xi$ See also Equations 17 to 19. Fixed parameters are not shown. 
Table 2

Resuls for the Effect Analysis.

\begin{tabular}{lccccc}
\hline & & \multicolumn{4}{c}{ Quantiles } \\
Coefficient & Mean & SD & $2.5 \%$ & $50 \%$ & $97.5 \%$ \\
\hline$\gamma_{10}$ & 1.80 & 0.93 & -0.09 & 1.82 & 3.69 \\
$\gamma_{11}$ & -2.10 & 0.71 & -3.55 & -2.11 & -0.62 \\
$\gamma_{12}$ & 0.37 & 0.12 & 0.11 & 0.37 & 0.62 \\
$E(\xi)$ & 2.85 & 0.07 & 2.71 & 2.85 & 2.98 \\
$E\left(\xi^{2}\right)$ & 9.33 & 0.41 & 8.55 & 9.32 & 10.15 \\
$E\left[\mathrm{CE}_{10}(\xi)\right]$ & -0.73 & 0.13 & -0.98 & -0.73 & -0.47 \\
$\mathrm{CE}_{10}(\xi=1)$ & 0.07 & 0.38 & -0.68 & 0.07 & 0.82 \\
$\mathrm{CE}_{10}(\xi=2)$ & -0.92 & 0.17 & -1.26 & -0.92 & -0.58 \\
$\mathrm{CE}_{10}(\xi=3)$ & -1.17 & 0.19 & -1.53 & -1.17 & -0.78 \\
$\mathrm{CE}_{10}(\xi=4)$ & -0.68 & 0.19 & -1.05 & -0.68 & -0.30 \\
\hline
\end{tabular}

Note. $\gamma_{10}, \gamma_{11}$, and $\gamma_{12}$ are parameters of the effect function, $\xi$ is the latent covariate self-efficacy, $\mathrm{CE}_{10}$ stands for the conditional effect function of training condition $X=1$ vs. control condition $X=0$. 


$$
X=0
$$

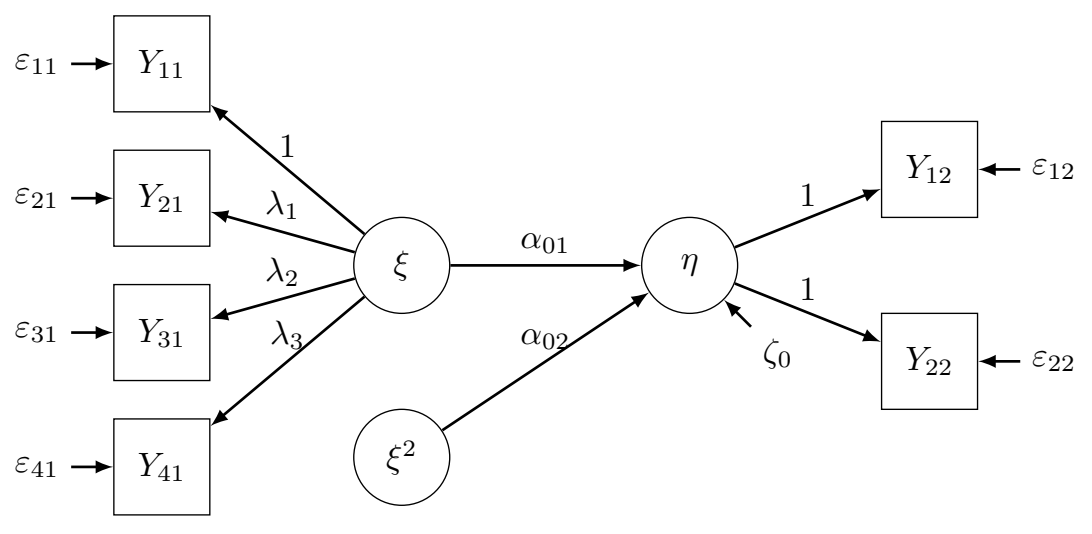

$$
X=1
$$

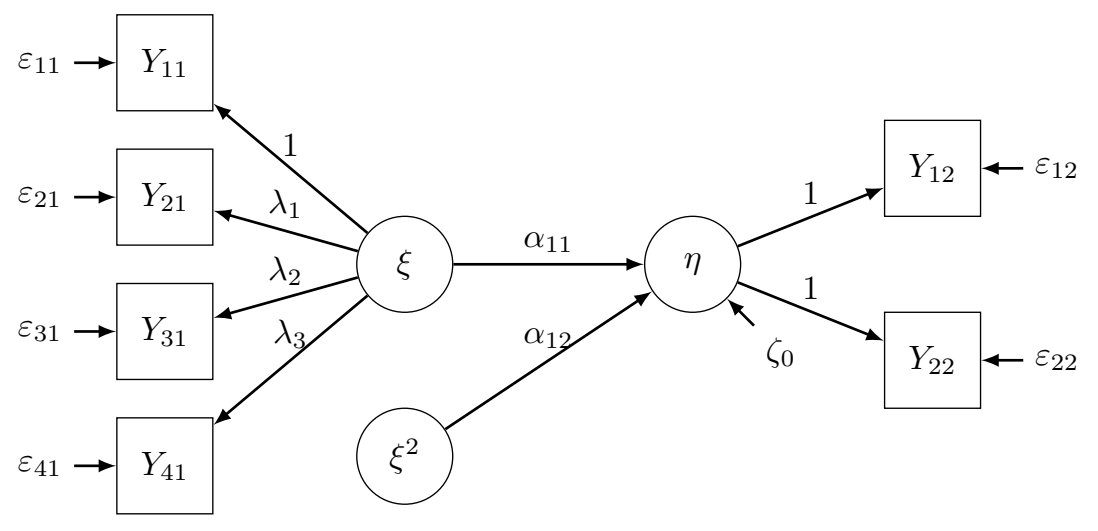

Figure 1. Path diagram for our illustrative example. It shows the two treatment group-specific regressions of the dependent variable $\eta$ on $\xi$ and $\xi^{2}$. 

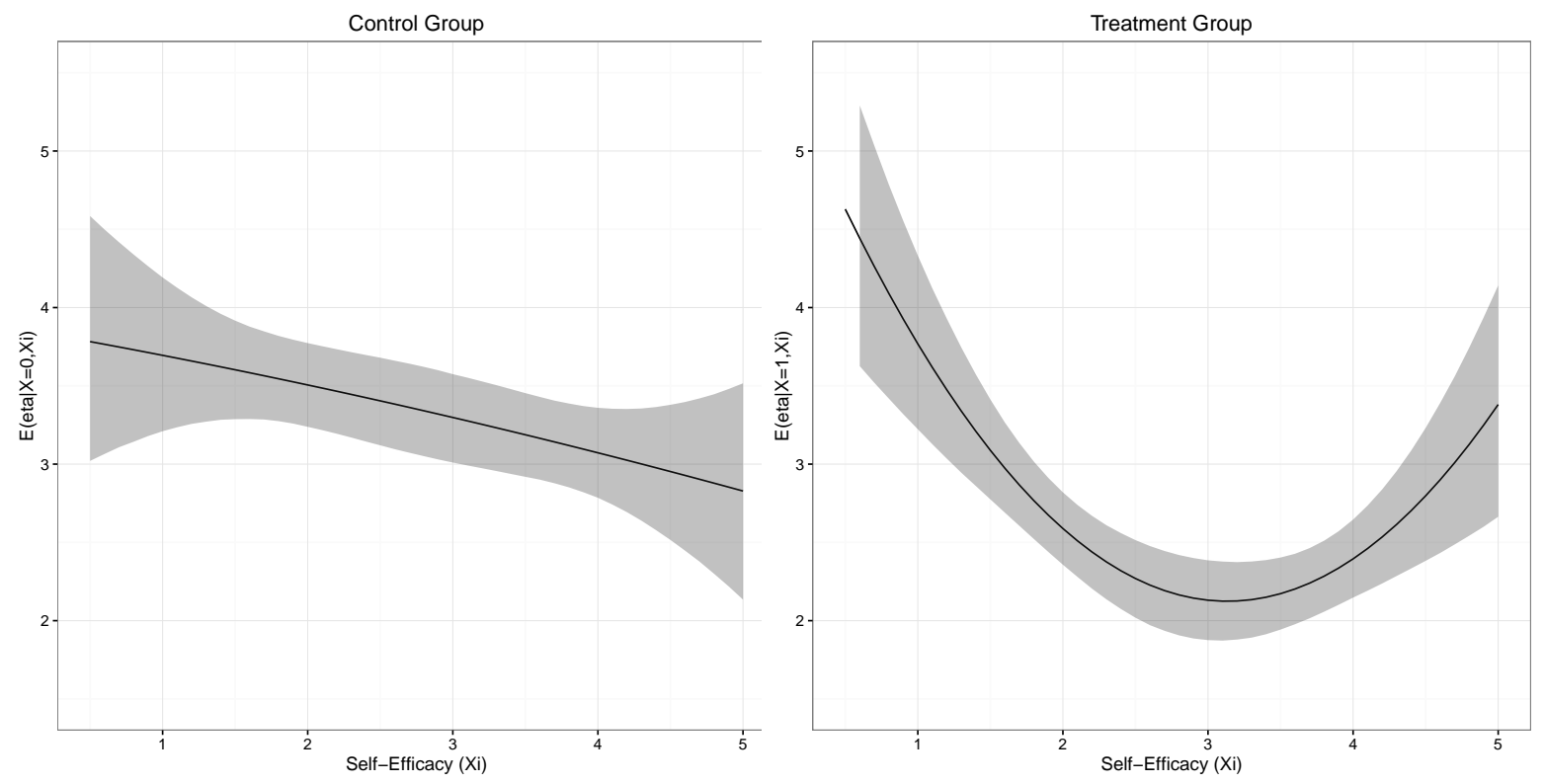

Figure 2. Plot of the group-specific regressions of $\eta$ on $\xi$ and $\xi^{2}$ in the control group (left-hand side) and the treatment group (right-hand side) with $95 \%$ credibility intervals. 


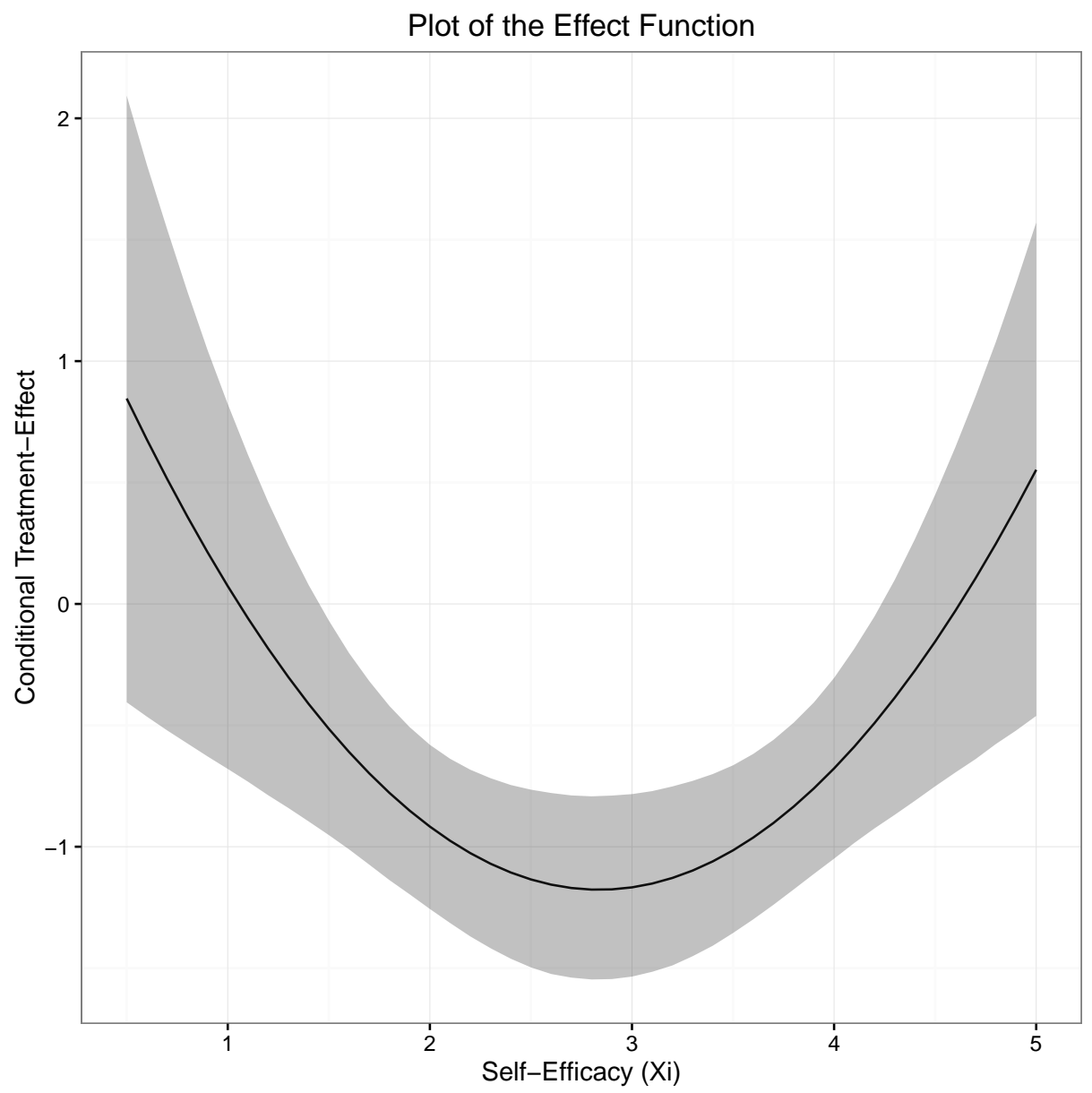

Figure 3. Plot of the conditional effect function $\mathrm{CE}_{10}(\xi)$ with $95 \%$ credibility interval. The $y$-axis shows effects of the treatment condition compared to the control condition. The $x$-axis shows values of the latent covariate $\xi$ (self-efficacy). 


\section{Appendix A}

Web Appendix: blavaan Code

We use blavaan (Merkle \& Rosseel, 2015) to generate the multigroup SEM and then modify the produced JAGS (Plummer, 2015) file to also include quadratic effects and non-normal latent predictors (see Appendix B). The blavaan code is:

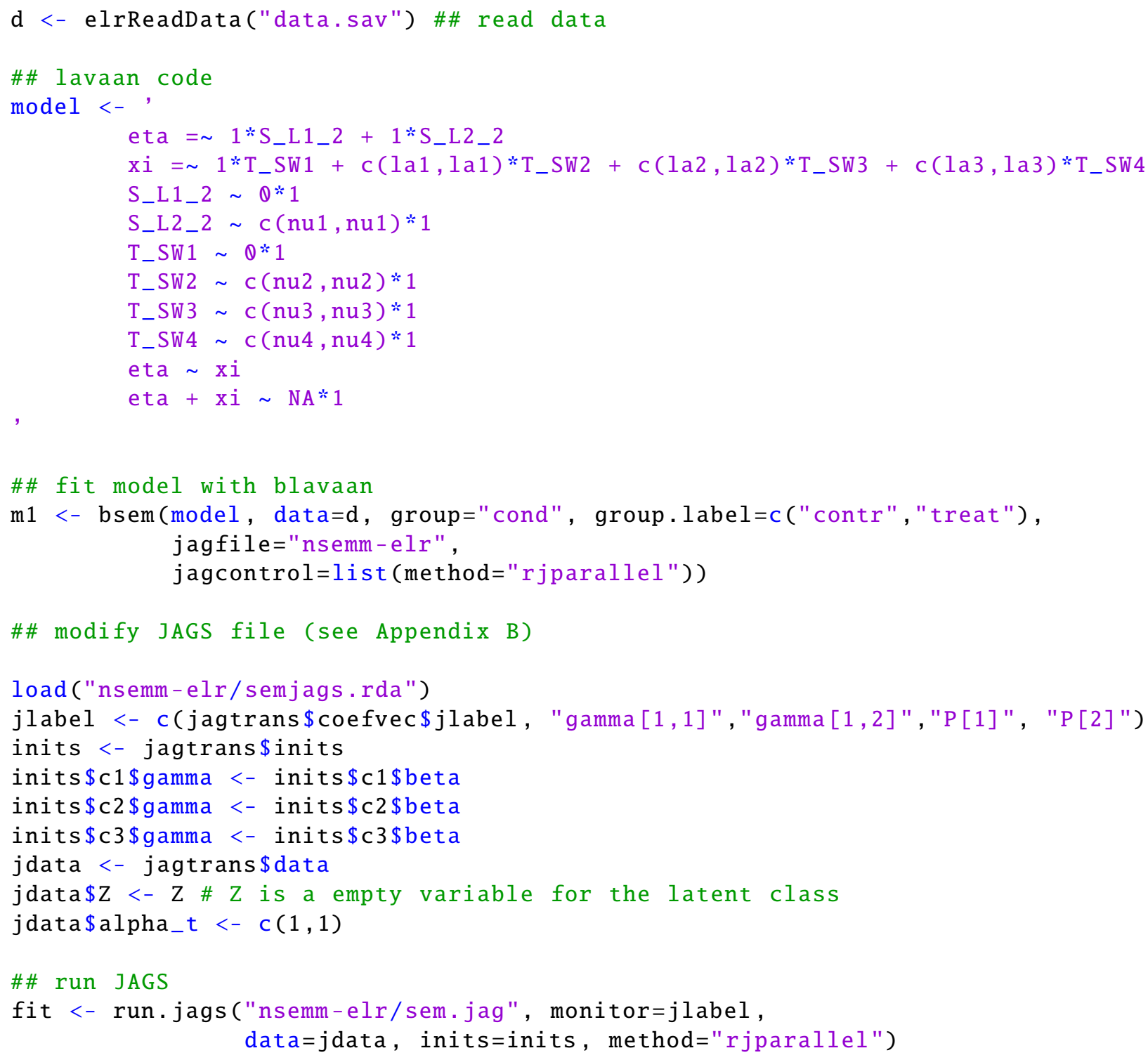




\section{Appendix B}

\section{Web Appendix: JAGS Code}

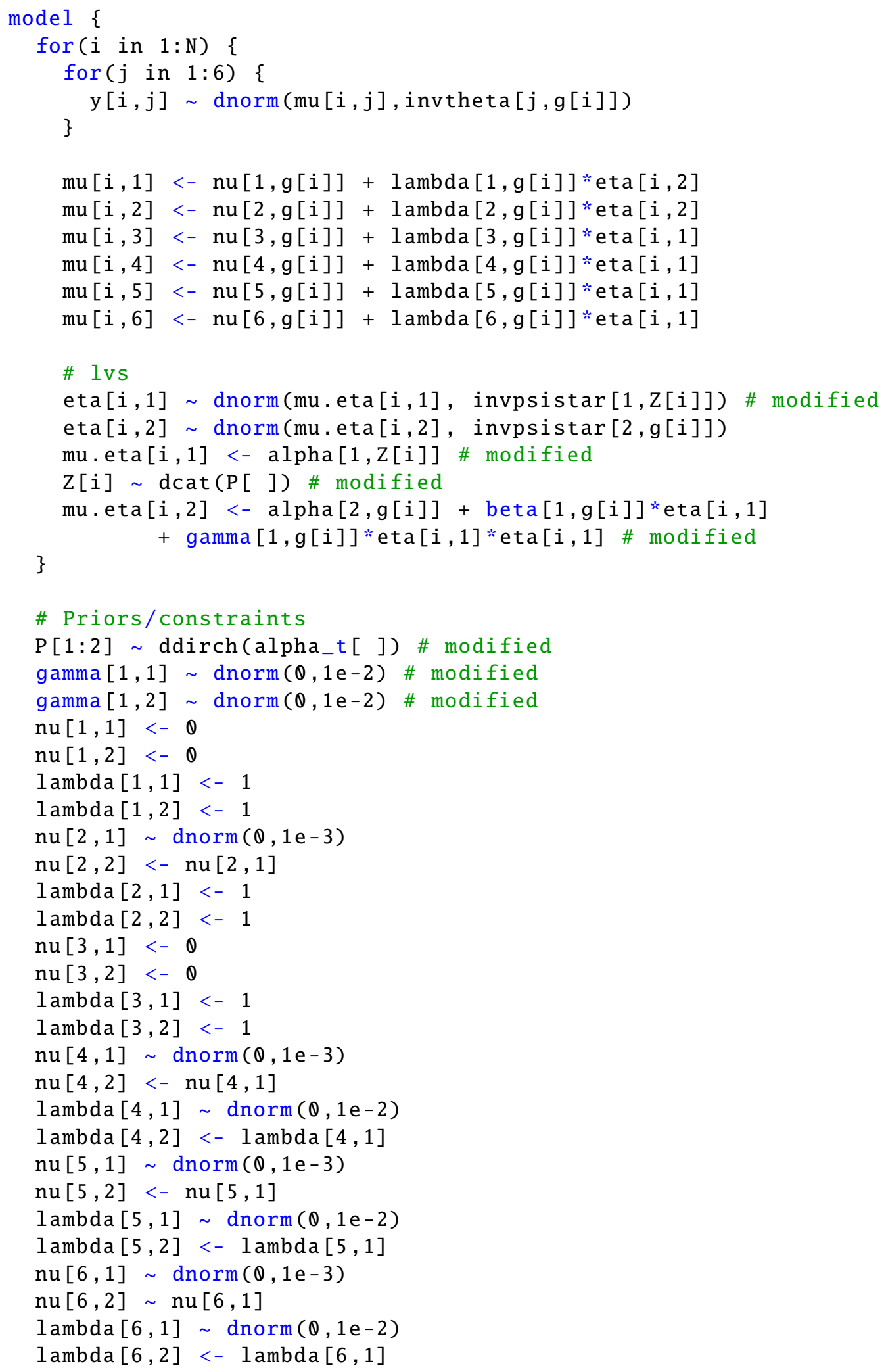




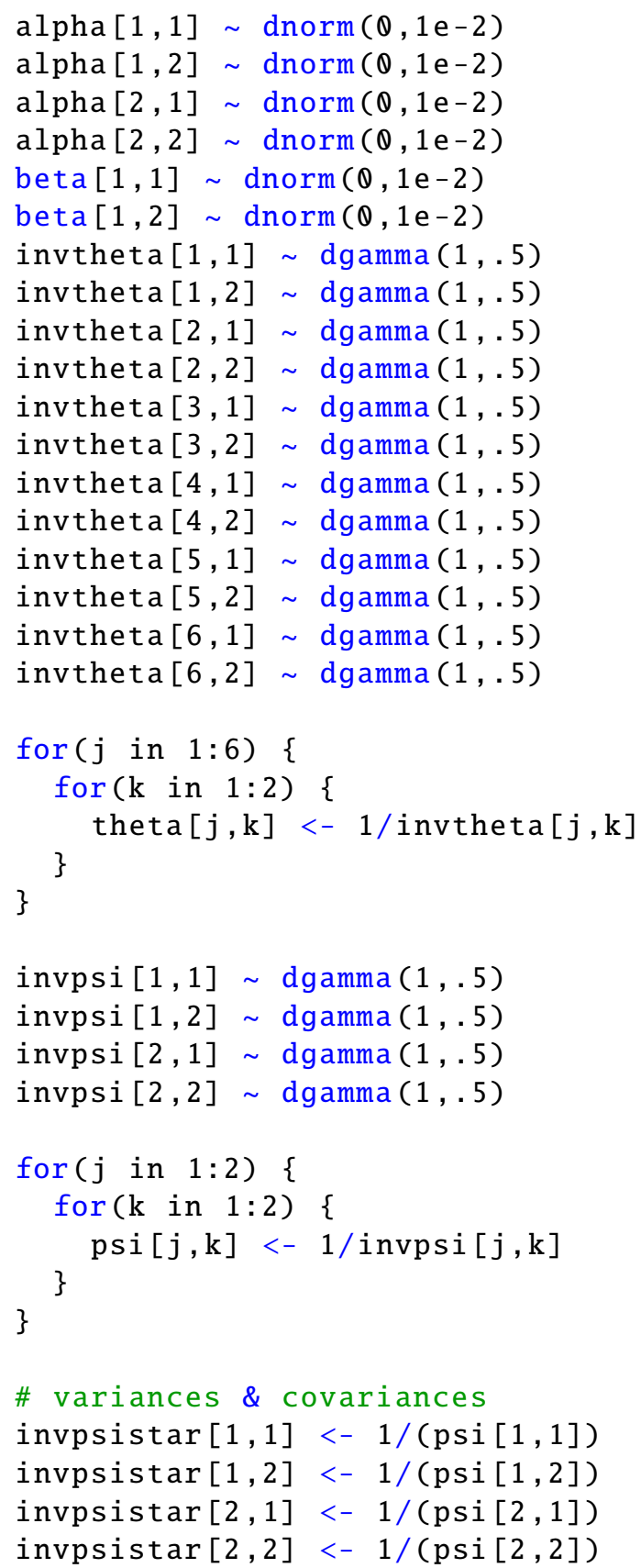




\section{Appendix C}

\section{Web Appendix: R Code Effect Computations}

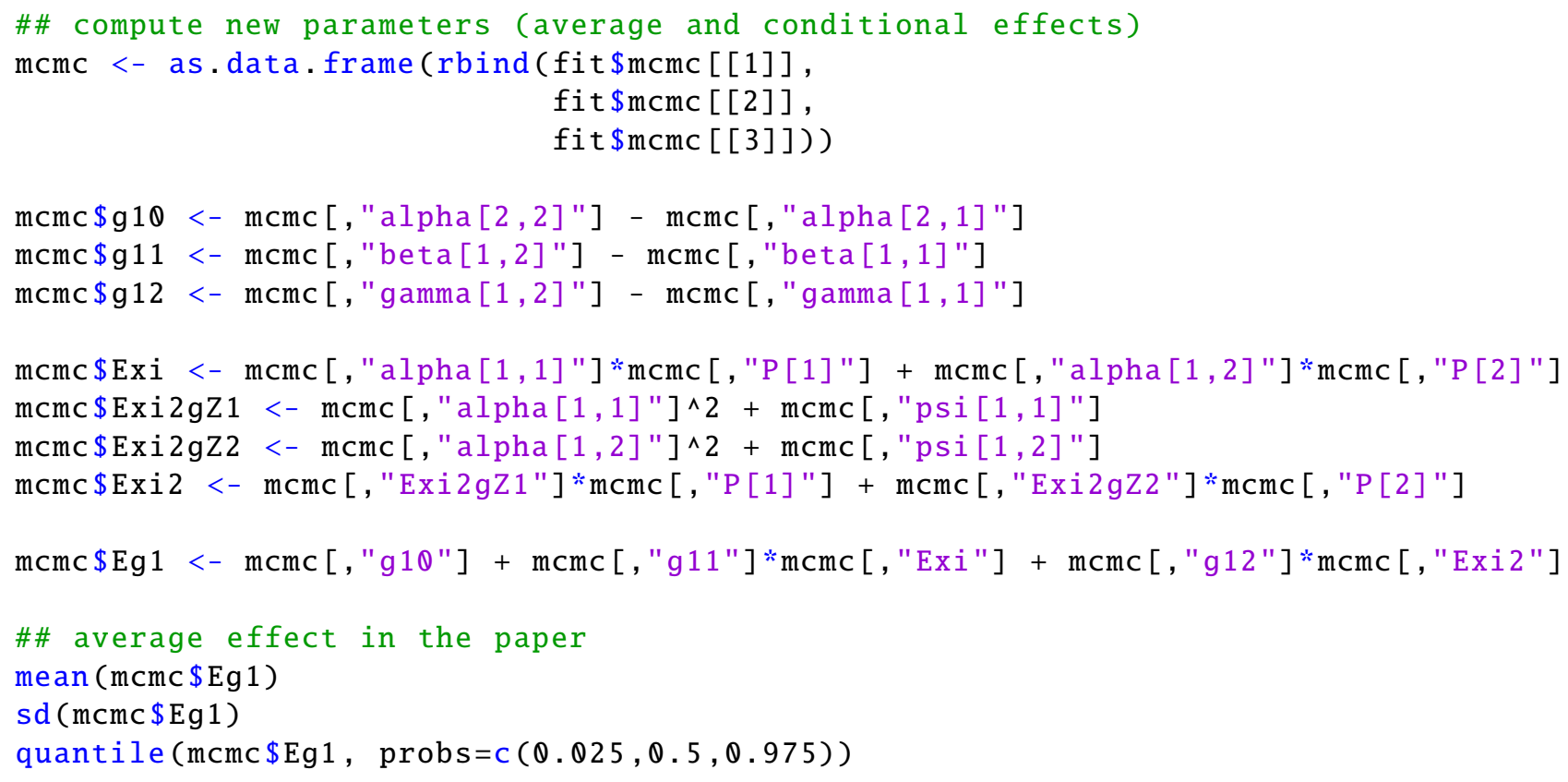

